\title{
PERFIL CLÍNICO E FUNCIONAL DO IDOSO NA ATENÇÃO PRIMÁRIA À SAÚDE EM BELO HORIZONTE
}

\section{CLINICAL AND FUNCTIONAL PROFILE OF THE ELDERLY IN PRIMARY HEALTH CARE IN BELO HORIZONTE}

\section{PERFIL CLÍNICO Y FUNCIONAL DEL ANCIANO EN LA ATENCIÓN PRIMARIA DE SALUD EN BELO HORIZONTE}

\author{
Thaynná Neres dos Santos ${ }^{1}$, Isabel Yovana Quispe Mendoza르, Silmar Maria da Silva ${ }^{3}$, Marcia Regina Martins Alvarenga ${ }^{4}$, Edmar \\ Geraldo Ribeiro 5
}

\section{RESUMO}

Objetivo: Analisar o perfil clínico e funcional do idoso assistido pela Atenção Primária de Saúde. Método: Trata-se de um estudo transversal, analítico descritivo, com abordagem quantitativa, realizado na Atenção Primária à Saúde, com 396 idosos. Aplicaram-se dois instrumentos: questionário sociodemográfico e o Índice de Vulnerabilidade Clínico Funcional 20. As análises estatísticas foram realizadas com o auxílio do software Statistical Package for the Social Sciences (versão 23), por meio dos testes Qui-Quadrado Simulado e Qui-Quadrado. Para variáveis qualitativas utilizaram-se frequências absoluta e relativa e para variáveis quantitativas medidas de tendência central, posição e dispersão. Resultados: Predomínio de idosos entre 60 e 74 anos (64,81\%); sexo feminino (65,40\%); casados (43,69\%); que mantiveram frequência escolar (83,59\%); católicos (70,33\%); sem cuidador (87,63\%); e renda média de $\mathrm{R} \$ 2194,95$ ( $\mathrm{DP}=4153,02$ ). O Índice de Vulnerabilidade Clínico Funcional- 20 apontou que 44,9\% dos idosos eram robustos, 42,4\% pré- frágeis, e $12,7 \%$ frágeis. Conclusão: Constatou-se maior prevalência de idosos robustos e recomendam-se ações voltadas à promoção da saúde e prevenção da fragilidade, além de incentivos na utilização de instrumentos de avaliação multidimensional da saúde da pessoa idosa.

Descritores: Envelhecimento; Saúde do Idoso; Enfermagem Geriátrica; Avaliação Geriátrica; Atenção Primária a Saúde.

\section{ABSTRACT}

Objective: To analyze the clinical and functional profile of the elderly assisted by Primary Health Care. Method: This is a crosssectional, analytical and descriptive study, with a quantitative approach, carried out in Primary Health Care, with 396 elderly people. Two instruments were applied: a sociodemographic questionnaire and the Functional Clinical Vulnerability Index 20. Statistical analyses were performed with the aid of software Statistical Package for the Social Sciences (version 23), using the Simulated ChiSquare and Chi-Square tests. For qualitative variables, absolute and relative frequencies were used and for quantitative variables, measures of central tendency, position and dispersion were used. Results: Predominance of elderly people between 60 and 74 years old (64.81\%); women (65.40\%); married (43.69\%); who maintained school attendance (83.59\%); Catholic (70.33\%); without caregiver (87.63\%); and average income of $\mathrm{R} \$ 2,194.95(\mathrm{SD}=4,153.02)$. The Clinical Functional Vulnerability Index- 20 showed that $44.9 \%$ of the elderly were robust, $42.4 \%$ pre-frail, and $12.7 \%$ frail. Conclusion: There is a higher prevalence of robust elderly people. Actions aimed at promoting health and preventing frailty are recommended, and the use of multidimensional assessment instruments for the health of the elderly is advised.

Descriptors: Aging; Health of the Elderly; Geriatric Nursing; Geriatric Assessment; Primary Health Care.

\section{RESUMEN}

Objetivo: Analizar el perfil clínico y funcional de los ancianos atendidos por la Atención Primaria de Salud. Método: Se trata de un estudio transversal, analítico y descriptivo, con abordaje cuantitativo, realizado en la Atención Primaria de Salud, con 396 ancianos. Se aplicaron dos instrumentos: cuestionario sociodemográfico y el Índice de Vulnerabilidad Clínica Funcional 20. Los análisis estadísticos se realizaron con la ayuda del software Statistical Package for the Social Sciences (versión 23), utilizando las pruebas de Chi-Cuadrado Simulado y Chi-Cuadrado. Para las variables cualitativas se utilizaron frecuencias absolutas y relativas, y para las cuantitativas se utilizaron medidas de tendencia central, posición y dispersión. Resultados: Predominio de ancianos entre 60 y 74 años (64,81\%); sexo femenino (65,40\%); casado (43,69\%); quién mantuvo la asistencia escolar $(83,59 \%)$; católicos $(70,33 \%)$; sin cuidador (87,63\%); e ingreso promedio de $\mathrm{R} \$ 2194,95(\mathrm{DE}=4153,02)$. El Índice de Vulnerabilidad Clínica Funcional-20 mostró que el $44,9 \%$ de los ancianos eran robustos, el $42,4 \%$ prefrágiles y el $12,7 \%$ frágiles. Conclusión: Existe un mayor predominio de ancianos robustos, y se recomiendan acciones orientadas a promover la salud y prevenir la fragilidad, así como incentivos en el uso de instrumentos de evaluación multidimensional para la salud de los ancianos.

Descriptores: Envejecimiento; Salud del Anciano; Enfermería Geriátrica; Evaluación Geriátrica; Atención Primaria de Salud.

${ }^{1}$ Enfermeira. Residente Multiprofissional em Saúde do Programa de Urgência e Emergência do Complexo Hospitalar de Contagem. ${ }^{2}$ Enfermeira. Doutora em Enfermagem. Professora Associada da Escola de Enfermagem da Universidade Federal de Minas Gerais. ${ }^{3}$ Enfermeira. Doutora em Enfermagem. Professora Adjunta da Escola de Enfermagem da Universidade Federal de Minas Gerais. ${ }^{4}$ Enfermeira. Pós-Doutorado em Enfermagem pela Universidade de São Paulo. Professora Adjunta do curso de Enfermagem da Universidade Estadual de Mato Grosso do Sul. ${ }^{5}$ Enfermeiro. Doutorando em Enfermagem pela Escola de Enfermagem da Universidade Federal de Minas Gerais

Como citar este artigo:

Santos TN, Mendoza IYQ, Silva SM, et al. Perfil clínico e funcional do idoso na atenção primária à saúde em Belo Horizonte. Revista de Enfermagem do Centro-Oeste Mineiro. 2020;10:e4038. [Access___]; Available in:__. DOI: http://doi.org/10.19175/recom.v10i0.4038 


\section{INTRODUÇÃO}

O processo do envelhecimento nas últimas décadas se destaca em nível mundial. No Brasil, esse fenômeno encontra-se associado, dentre outros aspectos, com a redução na taxa de fecundidade e o aumento na expectativa de vida. Em 2000, a população brasileira com mais de 60 anos era de 14,5 milhões de pessoas, representando aumento de $35,5 \%$ em relação aos 10,7 milhões em 1991. Atualmente, as projeções para o 2060, segundo o IBGE, é que o número de pessoas com mais de sessenta anos será, de aproximadamente, 82 milhões ${ }^{(1)}$.

O crescimento da população idosa traz novos desafios para o setor da saúde, porque os idosos apresentam necessidades específicas decorrentes da presença de Doenças Crônicas Não Transmissíveis (DCNT), o que acaba interferindo em sua qualidade de vida. Pessoas com 60 anos ou mais, em sua maioria, são acometidas por DCNT que requerem acompanhamento constante. Em alguns casos, gera um processo incapacitante no idoso, afetando o desempenho de suas atividades cotidianas: o comprometimento da capacidade clínica e funcional ${ }^{(2)}$.

A capacidade clínica e funcional avalia a aptidão, autonomia e a independência no cumprimento de atividades diretamente relacionadas com o autocuidado e com a participação social. Esse conceito caracteriza o potencial do idoso para decidir e atuar no seu cotidiano de maneira independente e autônoma ${ }^{(3)}$.

A redução da capacidade funcional do idoso pode desencadear o desenvolvimento da síndrome da fragilidade, definida como síndrome clínica geriátrica, de caráter multidimensional. A síndrome da fragilidade envolve um estado fisiológico que aumenta a vulnerabilidade a estressores pela diminuição das reservas fisiológicas e desregulação de múltiplos sistemas, associados à maior ocorrência de desfechos adversos como declínio da capacidade funcional, quedas, hospitalização, institucionalização e morte ${ }^{(4-5)}$.

Verifica-se que a Atenção Primária à Saúde (APS) é o primeiro contato do indivíduo na rede assistencial. Portanto o cuidado envolve reconhecer determinantes sociais e individuais, além das relações envolvidas para o cuidado nas dimensões biológicas, psicossociais e culturais. A avalição multidimensional do idoso na APS pode identificar e/ou evitar a perda da funcionalidade e, consequentemente, desenvolvimento da síndrome da fragilidade ${ }^{(6)}$.

A avaliação multidimensional é considerada o padrão-ouro para o manejo da síndrome da fragilidade no idoso, pois permite o reconhecimento das demandas biopsicossociais do indivíduo, ou seja, o diagnóstico clínico e funcional de suas condições de saúde agudas e/ou crônicas; essa avaliação permite reconhecer as incapacidades, tanto no que se refere à independência quanto à autonomia do idoso ${ }^{(5)}$.

Nesse sentido, a síndrome de fragilidade deve ser reconhecida como alvo para investigações e intervenções, tendo em vista o impacto sobre indivíduos idosos, suas famílias e a sociedade como um todo. Dessa forma, a identificação precoce das características dos diferentes grupos de idosos - robustos, em risco de fragilidade ou frágeis - pode ajudar a prevenir, retardar ou impedir a progressão da fragilidade, por meio de programas de cuidado e adequação dos serviços de saúde às novas demandas dessa população específica ${ }^{(7)}$.

Diante do exposto, a avaliação dos principais determinantes de saúde do idoso na APS se constitui em elemento fundamental, para a orientação dos profissionais de saúde na elaboração de plano de cuidados, indicação de intervenções multidisciplinares, identificação das dimensões clínicas e funcionais e direcionamento para consulta geriátrica especializada, com a finalidade de manter, melhorar a autonomia e independência do idoso. Este estudo tem como objetivo analisar o perfil clínico e funcional do idoso assistido pela APS na Regional Centro Sul de Belo Horizonte, Minas Gerais.

\section{MÉTODO}

Trata-se de um estudo transversal, analítico descritivo, norteado pela ferramenta Strengthening the Reporting of Observational Studies in Epidemiology (STROBE) ${ }^{(8)}$, com abordagem quantitativa, realizado em 12 Unidades Básicas de Saúde (UBS) da APS da regional Centro Sul de Belo Horizonte/Minas Gerais (BH/MG), Brasil. A região Centro Sul possui 49 bairros divididos em cinco territórios de gestão compartilhada. Em 2016, a região possuía uma população de 283.776 residentes na qual se identificava a maior faixa de pessoas acima de 60 anos da capital mineira, perfazendo 51.715 indivíduos ${ }^{(9)}$.

O cálculo da amostra foi realizado para que o estudo permitisse inferir seus resultados, para a 
população de idosos residentes na região Centro Sul de BH/MG. Para tanto, foi utilizado o método para estimação de proporções a populações finitas de forma aleatória, com alocação proporcional por UBS ${ }^{(10)}$. Considerando a margem de erro de $5 \%$, nível de significância de $5 \%$ e intervalo de confiança de $95 \%$ a amostra mínima necessária para o estudo foi de 381 idosos. E considerando o acréscimo de $20 \%$ para perdas, o tamanho amostral seria de 458 idosos. Assim, foram convidados 458 idosos, dentre os quais 396 aceitaram participar. A alocação da amostra proporcional por UBS e a amostra coletada encontram-se detalhadas na Figura 1.

Figura 1 - Tamanho da amostra estratificada por Unidade Básica de Saúde (UBS).

\begin{tabular}{cccccc}
\hline $\begin{array}{c}\text { Unidades Básicas de } \\
\text { Saúde }\end{array}$ & População & Proporção (\%) & Amostra & Amostra (20\%) & $\begin{array}{c}\text { Amostra } \\
\text { Coletada }\end{array}$ \\
A & & & 4 & 5 & 5 \\
B & 580 & 1,1 & 47 & 56 & 47 \\
C & 6.350 & 12,3 & 20 & 24 & 26 \\
D & 2.774 & 5,4 & 76 & 91 & 76 \\
E & 10.331 & 20,0 & 24 & 29 & 24 \\
F & 3.265 & 6,3 & 60 & 72 & 62 \\
G & 8.114 & 15,7 & 36 & 43 & 37 \\
H & 4.896 & 9,5 & 19 & 23 & 19 \\
I & 2.565 & 5,0 & 53 & 63 & 8 \\
Total & 1.110 & 2,1 & 4 & 5 & 53 \\
\hline
\end{tabular}

Fonte: Dados da pesquisa, 2018.

A amostragem foi probabilística e recrutada, por meio de seleção aleatória simples (sorteio), utilizando-se o programa Microsoft Excel (versão 2016). Inicialmente foi solicitada aos gerentes das UBS a lista dos idosos cadastrados na Estratégia Saúde da Família (ESF) e do Programa de Agentes Comunitários de Saúde (PACS). A lista dispunha de informações como: nome do usuário, número de prontuário, idade, data de nascimento, logradouro, telefone de contato, microárea em que reside e nome da mãe.

Os critérios de inclusão adotados foram idosos com idade igual ou superior a 60 anos, de ambos os sexos, que residiam na região Centro Sul de Belo Horizonte / Minas Gerais e estivessem devidamente cadastrados na Estratégia de Saúde da Família (ESF) e/ou no Programa de Agentes Comunitários de Saúde (PACS). Dentre os critérios de exclusão, consideraram-se os idosos que não compareceram à avaliação na data e horários agendados e que não possuíam telefone de contato na lista dos idosos cadastrados.

As avaliações foram agendadas previamente, via contato telefônico, realizado por um membro da equipe de pesquisa. Os dados foram coletados nas UBS, por um enfermeiro e colaboradores previamente treinados, sob a coordenação do pesquisador principal no período de janeiro a abril de 2018.

Seguidamente, procedeu-se com a avaliação, mediante a aplicação dos seguintes instrumentos: questionário sociodemográfico, semiestruturado, contendo as seguintes informações: sexo, idade, estado civil, moradia, escolaridade, religião, se possui cuidador (formal ou informal), renda familiar, dependentes da renda e profissão e instrumento que mede a condição clínica funcional do idoso - Índice de Vulnerabilidade Clínico Funcional-20 (IVCF-20). Cada avaliação teve duração média de 15 minutos. Realizou-se a coleta de segunda a sexta-feira, conforme disponibilidade do participante da pesquisa.

O IVCF-20 é um instrumento de triagem interdisciplinar, que contempla aspectos multidimensionais da condição de saúde dos indivíduos de 60 anos ou mais. Foi idealizado considerando a base conceitual de fragilidade como uma síndrome multidimensional e é constituído por 20 questões distribuídas em oito seções que contemplam diversos domínios e fatores relacionados à saúde. Cada seção possui uma pontuação específica que, no total, perfaz o valor máximo de 40 pontos, identificando a condição clínica funcional do idoso. Quanto maior a pontuação do IVCF-20, pior será a condição clínica funcional do idoso. Assim, ele é classificado como: robusto (0 a 06 pontos), em risco de fragilidade (07 a 14 pontos) e idoso frágil (15 pontos ou mais) $)^{(5)}$.

Em estudo que teve por finalidade avaliar a adequação do IVCF-20, no Centro de Referência ao 
Idoso (CRI) e na APS, os autores demonstraram que o instrumento é correlacionado positivamente com a Avaliação Geriátrica Ampla (AGA). Os resultados de validação pela curva Receiver Operating Characteristic (ROC) foi 0,903 (IC95\% 0,871-0,934) e o coeficiente alfa de Cronbach foi $0,74^{(5)}$.

Para a construção do banco de dados, foi utilizada a técnica de validação por dupla entrada no Programa Epi Info versão 3.5.1 (2008).

Na descrição das variáveis qualitativas da amostra, foram usadas as frequências absoluta e relativa e, para descrever as variáveis quantitativas, utilizaram-se medidas de tendência central, posição e dispersão. Para analisar as variáveis sociodemográficas em relação à condição de fragilidade, foram utilizados o teste Qui-Quadrado Simulado e Qui-Quadrado. Adotouse valor de significância de $p<0,05$. O software utilizado nas análises foi o SPSS (versão 23).

O presente estudo foi aprovado pelo Comitê de Ética em Pesquisa das instituições proponentes: Universidade Federal de Minas Gerais e Secretaria Municipal de Saúde da Prefeitura de Belo Horizonte/ Minas Gerais, por meio dos pareceres consubstanciados no CAAE: 75797617.6 .3001 .5140

CAAE: 75797617.6.0000.5149, respectivamente. O projeto atendeu os critérios da Resolução 466/12 e 510/2016 do Conselho Nacional de Saúde. Todos os participantes foram orientados sobre a pesquisa e, caso concordassem em participar, solicitou-se a assinatura do Termo de Consentimento Livre e Esclarecido (TCLE).

\section{RESULTADOS E DISCUSSÃO}

Ao serem avaliados pelo IVCF-20, o maior percentual $(44,9 \%)$ dos idosos foram classificados como robustos (Tabela 1). Resultado semelhante foi identificado em estudo realizado com idosos da comunidade em Minas Gerais ${ }^{(11)}$. Já em estudo ao Sul do Brasil, a prevalência de fragilidade foi de $17,7 \%$, idosos em risco de fragilidade que representaram $45,4 \%$ e $36,9 \%$ e foram considerados robustos. Em comparação ao sexo, a prevalência de fragilidade no sexo feminino foi de $18,0 \%$ e em risco de fragilidade de $47 \%$, já no sexo masculino foi de $17,1 \%$ frágeis e $42,9 \%$ em risco de fragilização(12).

Tabela 1 - Distribuição dos participantes segundo o Índice de Vulnerabilidade Clínico Funcional 20. Belo Horizonte, MG, Brasil, 2018 ( $n=396)$.

$\begin{array}{lll}\text { IVCF-20 N } & \text { N }\end{array}$

\begin{tabular}{lcc}
\hline Robusto & 178 & 44,9 \\
Risco de Fragilidade & 168 & 42,4 \\
Frágil & 50 & 12,7 \\
\hline Total & $\mathbf{3 9 6}$ & $\mathbf{1 0 0 , 0}$ \\
\hline \hline
\end{tabular}

Fonte: Dados da pesquisa, 2018.

Estudos sobre fragilidade e funcionalidade dos idosos destacam a importância do profissional de saúde estar apto a identificar precocemente sinais e sintomas da condição de risco de fragilidade do idoso, a partir de medidas específicas e individualizadas, com intervenções que busquem potencializar a sua qualidade de vida e evitar a instalação da síndrome de fragilidade ${ }^{(12)}$.

Em estudo realizado na Paraíba que utilizou - IVCF-20, os resultados mostraram que a maioria dos idosos eram frágeis ${ }^{(13)}$. A partir dos resultados do presente estudo, infere-se que os idosos estão em plena condição de realizar suas atividades, com autonomia e independência, isto é, ter um envelhecimento ativo. A finalidade do envelhecimento ativo é aumentar a expectativa de vida saudável e a qualidade de vida para todas as pessoas que estão envelhecendo. Ainda, a Organização Mundial de Saúde (OMS) destaca que os fatores culturais, de gênero, serviços sociais e de saúde, aspectos econômicos, aspectos sociais e ambiente físico, pessoais e comportamentais são determinantes do envelhecimento ativo ${ }^{(14-16)}$.

Menores percentuais de idosos frágeis foram encontrados no presente estudo. $O$ idoso frágil é aquele que apresenta declínio em sua capacidade funcional, não sendo capaz de gerir a sua vida com autonomia e independência. $\mathrm{Na}$ maioria das vezes, essa síndrome instala-se de forma rápida ou é agravada pela presença de doenças crônicas que aceleram o processo de perda de resistência aos estressores ${ }^{(17)}$. No entanto estudos mostram que a fragilidade é uma síndrome que pode ser revertida, desde que sejam tomadas ações eficazes que interrompam a progressão da síndrome ${ }^{(17)}$.

A adoção de um conceito multidimensional com os componentes físicos, psicológicos e sociais, além do conhecimento da heterogeneidade do envelhecimento e dos fatores que influenciam na qualidade de vida, é essencial para definir políticas e programas direcionados à pessoa idosa. Aqui destaca-se o cuidado integrado e continuado do idoso na APS ${ }^{(5)}$.

Assim, a utilização de um instrumento de triagem multidimensional da saúde do idoso na APS, como o IVCF-20, é de suma importância, pois 
poderá ajudar a estratificar o idoso e identificar quais as dimensões da saúde do idoso foram afetadas. Dessa maneira, implementem-se os planos de cuidado interdisciplinar, além de nortear os encaminhamentos de referência e contrarreferência a centros de referência do idoso(5).

A Tabela 2 apresenta a relação entre a condição de fragilidade avaliada pelo IVCF-20 com as variáveis sociodemográficas. A UBS C detém a maioria de idosos robustos e em risco de fragilidade e a UBS A apresenta mais idosos classificados como frágeis. Destaca-se que o sexo feminino predomina, significativamente, quanto aos três tipos de classificações de condição de fragilidade. No que se refere aos anos de estudo, ter menos escolaridade reflete/interfere ou está associado às condições de fragilidade. A maioria dos idosos não possuía cuidador.

Tabela 2 - Caracterização dos participantes segundo a condição de fragilidade. Belo Horizonte, MG, Brasil, 2018 ( $n=396)$.

\begin{tabular}{|c|c|c|c|c|c|c|c|c|}
\hline \multicolumn{2}{|c|}{ Variáveis\Condição de Fragilidade } & \multicolumn{2}{|c|}{ Idoso robusto } & \multicolumn{2}{|c|}{ Risco de fragilidade } & \multicolumn{2}{|c|}{ Idoso frágil } & \multirow[t]{2}{*}{ Valor-p } \\
\hline & & $\mathbf{N}$ & $\%$ & $\mathbf{N}$ & $\%$ & $\mathbf{N}$ & $\%$ & \\
\hline \multirow{10}{*}{ Unidade Básica de Saúde } & $A$ & 17 & 9,55 & 13 & 9,42 & 17 & 21,25 & \multirow{10}{*}{$0,002^{1}$} \\
\hline & B & 13 & 7,30 & 06 & 4,35 & 07 & 8,75 & \\
\hline & $\mathrm{C}$ & 35 & 19,66 & 31 & 22,46 & 10 & 12,50 & \\
\hline & $\mathrm{D}$ & 16 & 8,99 & 05 & 3,62 & 03 & 3,75 & \\
\hline & $E$ & 11 & 6,18 & 05 & 3,62 & 03 & 3,75 & \\
\hline & $\mathrm{F}$ & 21 & 11,80 & 25 & 18,12 & 16 & 20,00 & \\
\hline & G & 23 & 12,92 & 07 & 5,07 & 07 & 8,75 & \\
\hline & $\mathrm{H}$ & 14 & 7,87 & 30 & 21,74 & 09 & 11,25 & \\
\hline & 1 & 17 & 9,55 & 09 & 6,52 & 05 & 6,25 & \\
\hline & Outros (J, K, L) & 11 & 6,18 & 07 & 5,07 & 03 & 3,75 & \\
\hline \multirow{2}{*}{ Sexo } & Masculino & 76 & 42,70 & 44 & 31,88 & 17 & 21,25 & \multirow{2}{*}{$0,003^{2}$} \\
\hline & Feminino & 102 & 57,30 & 94 & 68,12 & 63 & 78,75 & \\
\hline \multirow{2}{*}{ Estado Civil } & Com companheiro & 82 & 46,07 & 55 & 39,86 & 36 & 45,00 & \multirow{2}{*}{$0,525^{2}$} \\
\hline & Sem companheiro & 96 & 53,93 & 83 & 60,14 & 44 & 55,00 & \\
\hline \multirow{2}{*}{ Casa Própria } & Sim & 146 & 82,02 & 113 & 81,88 & 61 & 76,25 & \multirow{2}{*}{$0,511^{2}$} \\
\hline & Não & 32 & 17,98 & 25 & 18,12 & 19 & 23,75 & \\
\hline \multirow{2}{*}{ Mora sozinho } & Sim & 34 & 19,10 & 20 & 14,49 & 07 & 8,75 & \multirow{2}{*}{$0,097^{2}$} \\
\hline & Não & 144 & 80,90 & 118 & 85,51 & 73 & 91,25 & \\
\hline \multirow{2}{*}{ Anos de Estudo } & $\leq 8$ anos de estudo & 106 & 60,57 & 97 & 72,39 & 61 & 78,21 & \multirow{2}{*}{$0,009^{2}$} \\
\hline & $>8$ anos de estudo & 69 & 39,43 & 37 & 27,61 & 17 & 21,79 & \\
\hline \multirow{4}{*}{ Religião } & Católico & 114 & 65,14 & 102 & 75,00 & 59 & 73,75 & \multirow{4}{*}{$0,285^{2}$} \\
\hline & Evangélico & 40 & 22,86 & 24 & 17,65 & 18 & 22,50 & \\
\hline & Outras & 09 & 5,14 & 04 & 2,94 & 02 & 2,50 & \\
\hline & Sem religião & 12 & 6,86 & 06 & 4,41 & 01 & 1,25 & \\
\hline \multirow{2}{*}{ Possui cuidador } & Sim & 03 & 1,69 & 16 & 11,59 & 30 & 37,50 & \multirow{2}{*}{$0,000^{2}$} \\
\hline & Não & 175 & 98,31 & 122 & 88,41 & 50 & 62,50 & \\
\hline \multirow{2}{*}{ Ocupação } & Aposentado & 159 & 90,34 & 127 & 92,70 & 72 & 91,14 & \multirow{2}{*}{$0,761^{2}$} \\
\hline & Outros & 17 & 9,66 & 10 & 7,30 & 07 & 8,86 & \\
\hline
\end{tabular}

Fonte: Dados da pesquisa, 2018.

Nota: 'Qui-Quadrado Simulado; ${ }^{2}$ Qui-Quadrado.

Ao relacionar os dados sociodemográficos com o IVCF-20, destaca-se associação significativa com a variável UBS. É importante destacar que as prefeituras utilizam o Índice de Vulnerabilidade da Saúde (IVS) como indicador para alocar os 
recursos humanos às áreas de maior risco de adoecimento e vulnerabilidade ${ }^{(9)}$.

Segundo o IVS, registrado pela Prefeitura de Belo Horizonte $(\mathrm{PBH})$, a população descrita nas UBS D, F, J e B, estão classificadas como baixo risco de vulnerabilidade, isto é, áreas com menor risco de adoecimento ${ }^{(9)}$. No entanto, quando avaliadas as respectivas UBS pelo IVCF-20, foram identificados idosos frágeis e com risco de fragilidade. Por outra parte, as UBS K e A, segundo o IVS, foram classificadas como áreas de risco elevado e muito elevado de vulnerabilidade; já no presente estudo, quando avaliados pelo IVCF-20, os idosos foram classificados como robustos.

As divergências identificadas podem justificar-se pela heterogeneidade existente nos idosos nas UBS e porque o IVS analisa as variáveis sociais, econômicas e de saneamento básico. Dessa forma, pode-se inferir que o IVS é um instrumento que avalia determinadas variáveis, que divergem com as variáveis contempladas pelo IVCF-20 - que avalia o estado clínico e funcional. Entretanto ressalta-se que o IVS e o IVCF-20 podem ser utilizados de forma associada, a fim de retratar as situações de vulnerabilidades e, consequentemente, fragilidade no idoso.

Com relação ao sexo, mostrou-se associação estatisticamente com o IVCF-20; estudos de coorte, de âmbito internacional, como dos Estados Unidos, França e Espanha, mostraram maior prevalência de fragilidade em idosos do sexo feminino(18). Estudo nacional também apresenta resultado semelhante: prevalência de $20,1 \%$ de idosos frágeis, $31,2 \%$ de idosos préfrágeis e, dentre os fatores associados, destacamse: sexo feminino ${ }^{(7)}$.

As Tabelas 3 e 4 apresentam a análise descritiva das variáveis demográficas e socioeconômicas qualitativas e quantitativas, respectivamente.

Tabela 3 - Análise descritiva das variáveis demográficas e socioeconômicas. Belo Horizonte, MG, Brasil, 2018 ( $n=396)$.

\begin{tabular}{llcc}
\hline \multicolumn{1}{c}{ Variáveis } & & $\mathbf{N}$ & $\%$ \\
\hline Sexo (n=396) & Masculino & 137 & 34,60 \\
& Feminino & 259 & 65,40 \\
Estado Civil & Solteiro & 94 & 23,74 \\
$(\mathrm{n}=396)$ & Casado/União & 173 & 43,69 \\
& Estável & 35 & 8,84 \\
& Separado/Divorciado & 94 & 23,74 \\
& Viúvo & & \\
Frequência & Sim & 331 & 83,59 \\
escolar (n=396) & Não & 65 & 16,41 \\
& & & \\
& Católico & 275 & 70,33 \\
& Evangélico & 82 & 20,97 \\
Religião & Espírita & 11 & 2,81 \\
(n=391) & Sem Religião & 15 & 3,84 \\
& Prefere não declarar & 03 & 0,77 \\
& Outros & 05 & 1,29 \\
& & & \\
Possui & Sim & 49 & 12,37 \\
cuidador & Não & 347 & 87,63 \\
(n=396) & & & \\
\hline \hline
\end{tabular}

Fonte: Dados da pesquisa, 2018

Tabela 4 - Análise descritiva das variáveis demográficas e socioeconômicas quantitativas. Belo Horizonte, MG, Brasil, 2018 ( $n=396)$.

\begin{tabular}{lllllllll}
\hline \hline Variáveis & N & Média & D.P. & Mínimo & 10 Q. & 20 Q. & 30 Q. & Máximo \\
\hline Anos de Estudo & 387 & 7,13 & 5,25 & 0,00 & 4,00 & 6,00 & 10,00 & 30,00 \\
Idade & 395 & 72,04 & 7,34 & 60,00 & 66,00 & 71,00 & 77,00 & 98,00 \\
Renda & 374 & 2194,95 & 4153,02 & 0,00 & 954,00 & 954,00 & 2000,00 & 60000,00 \\
No de dependentes da renda & 379 & 2,03 & 1,74 & 0,00 & 1,00 & 2,00 & 2,00 & 20,00 \\
\hline \hline
\end{tabular}

Fonte: Dados da pesquisa, 2018.

Nota: 1ㅇQ. - Primeiro Quartil / 2ㅇ Q. - Segundo Quartil / 3ㅇ‥ Terceiro Quartil / D.P - Desvio-Padrão.

Em pesquisa realizada ao Sul do Brasil, houve resultados semelhantes ao apresentado neste estudo, quando se identifica predominância das variáveis: sexo feminino $(68,4 \%)$, casados $(53,8 \%)$ e baixa renda (entre 1 e 2 salários mínimos com 2 dependentes) $)^{(19)}$.

Outro fator associado ao IVCF-20 são os anos de estudo, a maior parte dos participantes teve menos de oito anos de estudo. Esses dados são superiores à média de anos de estudo encontrados por outros autores em que a média de anos de estudo foi de 2,79 anos estudados ${ }^{(13)}$.
Os anos de estudo são considerados um determinante social que está relacionado com o acesso aos meios de cuidado e potencializa a vulnerabilidade do idoso de ser acometido por doenças, especialmente, as $\operatorname{DCNT}^{(13,20)}$.

A variável cuidador formal e/ou informal também foi associada com o IVCF-20. A maioria dos idosos robustos prefere não possuir cuidador, o que pode ser devido ao predomínio de idosos jovens e que ainda possuem cônjuge. Muitos idosos, também, são acompanhados de filhos e/ou familiares. Considera-se, portanto que a 
presença familiar e o papel da família no cuidado ao idoso são fundamentais para a sua estabilidade emocional e consequente melhoria da qualidade de vida. Entretanto identifica-se uma parcela significativa de idosos pré-frágeis e frágeis sem cuidador, o que acarreta no comprometimento da capacidade em reagir a situações adversas, refletindo no entorno sociocultural e na qualidade de vida do idoso ${ }^{(21-23)}$.

No presente estudo, no que se refere aos dados sociodemográficos, os resultados são condizentes com o descrito na literatura, isto é, houve predomínio de idosos entre 60 a 74 anos, com feminização da velhice, casados ou com união estável(7).

Uma variável que chama a atenção são os valores da renda que variaram significativamente, desde idosos sem renda até idosos com renda aproximada de $\mathrm{R} \$$ 60.000,00; com valor da mediana de $\mathrm{R} \$ \mathbf{9 5 4 , 0 0}$. Esses resultados demonstram a desigualdade na distribuição de renda em que o estudo foi realizado, pois a região conta com bairros tradicionais considerados de alto padrão, até vilas e conjunto de aglomerados.

Segundo Pesquisa Nacional por Amostra de Domicílios (PNAD -2018), o Brasil tem percentuais de distribuição de renda divergentes quando analisadas as cinco regiões. Dentre as categorias sobre rendimento proveniente de outras fontes, o item aposentadoria ou pensão é o mais elevado. Há desigualdade de rendimento também quando analisados os gêneros e cor/raça, e os valores mais elevados da renda familiar per capita dos idosos, bem como da população como um todo, estão concentrados no topo da pirâmide da população ${ }^{(24)}$.

A partir do exposto, pode-se considerar que a escolaridade e a renda familiar são determinantes sociais que têm relação com o estilo e qualidade de vida dos indivíduos ${ }^{(12)}$.

Outra variável que vale a pena destacar é o número de dependentes por idoso, identificou-se um mínimo de dois dependentes e máximo de 20, para um mesmo idoso. Cada vez mais o idoso torna-se novamente o chefe da família, contribuindo financeiramente com sua aposentadoria. Esse fenômeno de liderar financeiramente a família se deve aos altos índices de desemprego, nascimento de filhos fora do casamento e divórcios de suas próximas gerações. A desigualdade social do Brasil também está relacionada com número de dependentes da renda, o que impacta na saúde do idoso, com declínio das condições que sustentam sua qualidade de vida ${ }^{(25)}$.

Os resultados do presente estudo salientam contribuições significativas para o conhecimento gerontológico, em especial, para a enfermagem na APS da regional Centro Sul de Belo Horizonte, proporcionando assim subsídio científico para que as equipes interdisciplinares realizem um planejamento da assistência aos idosos. Vale ressaltar que a enfermagem tende a promover ações de promoção à saúde, por meio da avaliação clínica e funcional do idoso, visando ao envelhecimento ativo e melhor qualidade de vida. O uso de ferramentas de baixo custo, para a preservação da independência funcional, assim como à redução de eventos adversos como a institucionalização e mortalidade precoce, devem ser implementados pelo enfermeiro da APS.

Quanto aos avanços para a área de enfermagem, envolvem a produção e o aprimoramento do cuidado gerontológico pautado na adoção de intervenções que venham a repercutir positivamente para essa população.

Como limitação do estudo, destaca-se: a restrição apenas a uma regional específica de Belo Horizonte, não conseguindo estendê-lo a toda a cidade.

\section{CONCLUSÃO}

O presente estudo evidenciou maior prevalência de idosos robustos (44,9\%), considerados com baixa vulnerabilidade clínicofuncional, seguida de idosos em risco de fragilidade $(42,4 \%)$ e idosos frágeis $(12,7 \%)$. Recomenda-se a utilização de instrumentos de avaliação multidimensional da saúde da pessoa idosa, de forma a subsidiar ações em saúde na APS e gerar novas políticas públicas específicas para a população acima de 60 anos.

A fragilidade influi na qualidade de vida da pessoa idosa, gerando ou potencializando incapacidades que podem repercutir de forma negativa à pessoa na condição frágil e para a família. Dessa forma, faz-se necessário analisar o perfil da população idosa assistida na APS para identificar precocemente situações que podem gerar a síndrome de fragilidade e conduzir as ações voltadas para cada grupo específico. O trabalho multiprofissional e interdisciplinar é fundamental nesse processo, visto que atua pela busca ativa e identificação da população do território, além dos cuidados em saúde.

Por fim, é de suma importância que equipes da APS desenvolvam ações de recuperação e 
reabilitação da funcionalidade, pela promoção da saúde e prevenção da vulnerabilidade clínicofuncional, protelando o desenvolvimento da fragilidade e complicações nos idosos.

\section{REFERÊNCIAS}

1- Instituto Brasileiro de Geografia e Estatística. Projeções da população do Brasil e unidades da Federação por sexo e idade: 2010-2060. Brasília: IBGE; 2020 [citado em 15 jul 2020]. Acesso em: https://www.ibge.gov.br/estatisticas/sociais/pop ulacao/9109-projecao-dapopulacao.html?=\&t=resultados

2- Souza MAH, Porto EF, Souza EL, Silva KI. Perfil do estilo de vida de longevos. Rev Bras Geriatr Gerontol. 2016;19(5):819-26. DOI: 10.1590/1809$\underline{98232016019.150224}$

3- Carneiro JA, Ramos GC, Barbosa AT, Mendonça JM, Costa FM, Caldeira AP. Prevalence and factors associated with frailty in non institutionalized older adults. Rev Bras Enferm. 2016;69(3):435-42. DOI: 10.1590/0034-7167.2016690304i

4- Maciel GMC, Santos RS, Santos TM, Menezes RMP, Vitor AF, Lira ALBC. Avaliação da fragilidade no idoso pelo enfermeiro: Revisão integrativa. Rev Enferm Cent-Oeste Min. 2016;6(3):2430-38. DOI: 10.19175/recom.v6i3.1010

5- Moraes EN, Carmo JA, Lanna FM, Azevedo RS, Machado CJ, Romero DEM. Clinical-Functional Vulnerability Index-20 (IVCF-20): Rapid recognition of frail older adults. Rev Saúde Pública 2016; 50:81. DOI: 10.1590/s1518$\underline{8787.2016050006963}$

6- Pereira LSM. O Cuidado ao idoso frágil na Atenção Primária a Saúde: Programa Mais Vida [dissertação]. Belo Horizonte: Universidade Federal de Minas Gerais; 2017.

7- Maia LC, Colares TFB, Moraes EN, Costa SM, Caldeira AP. Robust older adults in primary care: Factors associated with successful aging. Rev Saúde Pública 2020;54(35):1-11. DOI: 10.11606/s1518-8787.2020054001735

8- STROBE Statement-checklist of items that should be included in reports of observational studies. Equator Network 2020 [cited 2020 July 1]. Available in: https://www.equatornetwork.org/wp-

content/uploads/2015/10/STROBE checklist v4 combined.pdf

9- Secretaria de Estado de Saúde de Minas Gerais. Região Centro Sul 2016 [citado em 10 out 2020]. Acesso em: http://www.saude.mg.gov.br/

10- Bolfarine $H$, Bussab WO. Elementos de amostragem. São Paulo: Blucher; 2005.
11- Vieira RA, Guerra RO, Giacomin KC, Vasconcelos KSS, Andrade ACS, Pereira LSM, et al. Prevalência de fragilidade e fatores associados em idosos comunitários de Belo Horizonte, Minas Gerais, Brasil: Dados do estudo FIBRA. Cad Saúde Pública 2013 [citado em 10 out 2020]; 29(8):163143. DOI: $10.1590 / 0102-311 \times 00126312$

12- Gross CB, Kolankiewicz ACB, Schmidt CR, Berlezi EM. Frailty levels of elderly people and their association with sociodemographic characteristics. Acta Paul Enferm. 2018;31(2):20916. DOI: $10.1590 / 1982-0194201800030$

13- Alexandrino A, Cruz EKL, Medeiros PYD, Oliveira CBS, Araújo DS, Nogueira MF. Evaluation of the clinical-functional vulnerability index in older adults. Rev Bras Geriatr Gerontol. 2019;22(6): e190222. DOI: 10.1590/1981$\underline{22562019022.190222}$

14- Organização Mundial da Saúde (OMS). Relatório mundial de envelhecimento e saúde. Organização Genebra: OMS; 2015.

15- Gonçalves CD. Envelhecimento bem sucedido, envelhecimento produtivo e envelhecimento ativo: reflexões. Estud. interdiscipl. envelhec. [internet]. 2015 [citado em 5 out. 2020]. 20(2): 645-57. Disponível em: https://seer.ufrgs.br/RevEnvelhecer/article/view/ 49428/35463.

16- Massi G, Wosiacki FT, Guarinello AC, Lacerda ABM, Carvalho TP, Wanderbrooke AC, Cairo NG, Lima RR. Envelhecimento ativo: um relato de pesquisa-intervenção. Rev. CEFAC [internet]. 2018 Fev [citado em 5 out. 2020]; 20(1):5-12. Disponível em: http://www.scielo.br/scielo.php?script=sci arttex t\&pid=S1516-18462018000100005\&lng=en. doi: 10.1590/1982-0216201820113017.

17- Duarte YAO, Nunes DP, Andrade FB, Corona LP, Brito TRP, Santos JLF, Lebrão ML. Frailty in older adults in the city of São Paulo: Prevalence and associated factors. Rev. bras. Epidemiol. [internet]. 2018 [citado em 5 jun 2020]; 21(2): e180021. Disponível em: http://www.scielo.br/scielo.php?script=sci arttex t\&pid=S1415-790X2018000300418\&lng=en. doi: 10.1590/1980-549720180021.supl.2.

18- Mello AC, Engstrom EM, Alves LC. Healthrelated and socio-demographic factors associated with frailty in the elderly: A systematic literature review. Cad Saúde Pública 2014;30(6):1-25. DOI: 10.1590/0102-311X00148213

19- Luz EP, Dallepiane LB, Kirchner RM, Silva LAA. Perfil sociodemográfico e de hábitos de vida da população idosa de um município da região norte 
do Rio Grande do Sul, Brasil Rev Bras Geriatr Gerontol. 2014;17(2):303-14. DOI: 10.1590/S1809-98232014000200008

20- Matos AIP, Mourao I, Coelho I. Interação entre a idade, escolaridade, tempo de institucionalização e exercício físico na função cognitiva e depressão em idosos. Motri. 2016;12(2):38-47. DOI: 10.6063/motricidade.6805 21- Rabelo DF, Neri AL. Avaliação das relações familiares por idosos com diferentes condições sociodemograficas e de saúde. Psico-USF 2016;21(3):663-75. DOI: 10.1590/141382712016210318

22- Lino VTS, Rodrigues NCP, Camacho LAB, O'Dwyer G, Lima IS, Andrade MKN, et al. Prevalência de sobrecarga e respectivos fatores associados em cuidadores de idosos dependentes, em uma região pobre do Rio de Janeiro, Brasil. Cad Saúde Pública 2016;32(6): e00060115. DOI: 10.1590/0102-311X00060115

23- Labegalini CMG, Nogueira IS, Moretti AZP, Carreira L, Baldissera VDA. Demandas educativas de cuidadores familiares de idosos dependentes. Rev Enferm Cent-Oeste Min. 2016;1(6):19942008. DOI: $10.19175 /$ recom.v0i0.1129

24- Instituto Brasileiro de Geografia e Estatística. Rendimento de todas as fontes 2017. Brasília: PNAD; 2018.

25- Barros MBA, Goldbaum M. Challenges of aging in the context of social inequalities. Rev Saúde Pública 2018;52(supl 2):S1-3. DOI: 10.11606/s1518-8787.201805200supl2ed

Nota:

Artigo da Dissertação de Mestrado intitulada "Análise Psicométrica do Índice de Vulnerabilidade Clínica Funcional IVCF-20", do Programa de Pós-Graduação em Enfermagem da Escola de Enfermagem da Universidade Federal de Minas Gerais.

Recebido em: $16 / 10 / 2020$

Aprovado em: $14 / 12 / 2020$

Endereço de correspondência:

Edmar Geraldo Ribeiro

Av. Alfredo Balena, 190, sala 230. Santa Efigênia, Belo Horizonte, MG, Brasil. CEP: 30130-100.

E-mail: edmargribeiro@gmail.com 